Cite this: Phys. Chem. Chem. Phys., 2013, 15, 18694

Received 21st May 2013, Accepted 6th September 2013

DOI: $10.1039 / c 3 c p 52146 j$

www.rsc.org/pccp

\title{
Molecular structure and hydrogen bonding in pure liquid ethylene glycol and ethylene glycol-water mixtures studied using NIR spectroscopy
}

\begin{abstract}
Yujing Chen, ${ }^{a b}$ Yukihiro Ozaki ${ }^{\mathrm{b}}$ and Mirosław A. Czarnecki*c
The molecular structure and hydrogen bonding of ethylene glycol (EG) and EG-water mixtures in the liquid phase were studied by using near-infrared (NIR) spectroscopy. The spectra were evaluated using a two-dimensional (2D) correlation approach, moving-window 2D correlation analysis and chemometric methods. The minor changes for the $\mathrm{CH}$ stretching bands indicate that the structures of pure liquid EG and EG-water mixtures are determined by the intermolecular hydrogen bonding through the $\mathrm{OH}$ groups. The analysis of the $\nu_{2}+\nu_{3}$ combination band of water reveals that in EG-rich solutions the molecules of water are predominantly bonded with two molecules of EG and this cooperative hydrogen bonding is stronger than that in bulk water. Further increase in the water content leads to formation of small water clusters around $\mathrm{OH}$ groups of EG. Comparing results for the binary mixtures of water with different organic solvents one can conclude that the total amount and distribution of the polar groups are the most important factors determining the solubility of water in the organic phase. The distribution of these groups depends on the length and structure of the hydrocarbon chain. Due to high population and relatively uniform distribution of the $\mathrm{OH}$ groups of EG water has unlimited solubility in liquid EG.
\end{abstract}

\section{Introduction}

Binary mixtures of EG with water form a glassy state even at relatively low concentrations of EG, preventing the crystallization of ice below $0{ }^{\circ} \mathrm{C}$. Therefore, EG is one of the most important cryoprotectants, and it is widely used in technology and medicine. EG is also a model system for studies of the competition between intermolecular and intramolecular hydrogen bonding. Fig. 1 displays two conformers of EG, with (a) and without (b) intramolecular hydrogen bonding. As can be seen, due to the significant strain this bonding is expected to be very weak. Therefore, the presence of intramolecular hydrogen bonding in vicinal diols is still a subject of debate. Several research groups claim that the $50-100 \mathrm{~cm}^{-1}$ red-shift in the vibrational spectra of vicinal diols may be generated by $\sigma \Rightarrow \sigma^{*}$ hyperconjugative effects ${ }^{1,2}$ and the conformers of EG do not present any bond path assignable to an intramolecular hydrogen bonding. ${ }^{3}$ X-ray measurements evidence that crystalline EG does not have intramolecular hydrogen bonding, and the crystal structure is stabilized by intermolecular $\mathrm{O}-\mathrm{H} \cdots \mathrm{O}$ and

${ }^{a}$ School of Chemistry and Chemical Engineering,

South China University of Technology, Guangzhou 510640, China

${ }^{b}$ School of Science and Technology, Kwansei Gakuin University, Sanda, Hyogo 669-1337, Japan

${ }^{c}$ Faculty of Chemistry, University of Wrocław, F. Joliot-Curie 14, 50-383 Wrockaw,

Poland. E-mail: miroslaw.czarnecki@chem.uni.wroc.pl; Fax: +48-71-3282348 (a)

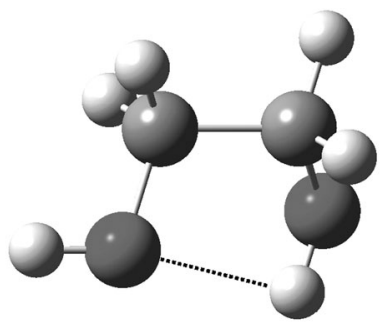

(b)

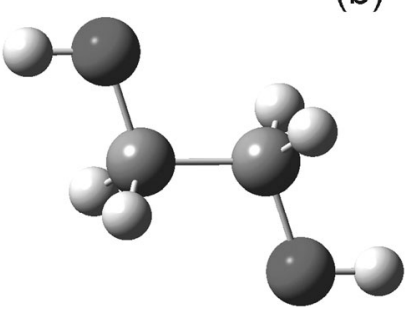

Fig. 1 Optimized structures of EG with (a) and without (b) intramolecular hydrogen bonding obtained from DFT calculations.

C-H. O interactions. ${ }^{4}$ A molecular dynamics (MD) study of EG-water mixtures shows that the intramolecular hydrogen bonds in EG molecules are hardly noticed, and in the solutions (molalities from 1 to 5 ) intermolecular hydrogen bonding 
between water molecules dominate. ${ }^{5}$ Also Raman spectra in the $\mathrm{OH}$ stretching band region do not reveal significant intramolecular hydrogen bond formation in pure liquid EG. ${ }^{6}$

In contrast, vapour-phase overtone spectra of EG are dominated by two conformers that have weak intramolecular hydrogen bonding with red-shifts of 30 and $45 \mathrm{~cm}^{-1}$. $^{7} \mathrm{Ab}$ initio conformational analysis shows that the lowest energy conformers of EG have gauche OCCO arrangements and are stabilized by intramolecular hydrogen bonding. ${ }^{8-11}$ However, in the lowest energy $1: 1$ complex with water this bonding is disrupted, and EG forms two intermolecular hydrogen bonds of equal strength. ${ }^{10,11}$ DFT studies coupled with many-body analysis in EG- $\left(\mathrm{H}_{2} \mathrm{O}\right)_{n}(n=1-3)$ complexes evidence that the structure in which a water molecule bridges two $\mathrm{OH}$ groups of EG is more stable than the other structures. $^{12}$ The quantum chemical conformational analysis reveals that the population of EG molecules with no intramolecular hydrogen bonding increases from 17 to $25 \%$ upon solvation. ${ }^{13}$ In contrast, an ATR-IR spectroscopic study of neat EG proves that the entire population of $\mathrm{OH}$ groups is involved in the inter- and intramolecular hydrogen bonding. ${ }^{14}$ The temperature raise leads to an increase in the population of the dimeric species at the expense of the species with the intramolecular hydrogen bonding. Recent $a b$ initio and DFT studies have shown that EG-water interaction is predominant over the EG-EG and waterwater interactions. ${ }^{15}$ It has been found that the second molecule of water does not create a hydrogen bond with the first molecule of water, but breaks a hydrogen bond between two EG molecules. The second hydrogen bond in the EG dimer remains intact. The water-water interaction occurs after addition of three or more molecules of water.

Though the presence of the intramolecular hydrogen bond in vicinal diols has not been convincingly evidenced as yet, this bond plays an important role in the stabilization of the structure of 1,3- and 1,4-diols. ${ }^{16,17}$ Our recent studies did not reveal the presence of the intramolecular hydrogen bond in diluted $\mathrm{CCl}_{4}$ solutions of 1,2-propanediol (12PD), while it was clearly manifested in the spectra of 1,3-propanediol (13PD) solutions. ${ }^{18}$ An increase in the concentration of 13PD shifts the equilibrium towards the formation of the intermolecular hydrogen bonding at the expense of the intramolecular one. The addition of water leads to faster thermal disruption of the associates of propanediols in the liquid phase. This means that the creation of hydrogen bonding between diol and water weakens the diol-diol interactions, as observed for EG-water complexes. ${ }^{15}$ The optimized structures of EG-water complexes shown in ref. 15 (Fig. 2) have at least one free $\mathrm{OH}$ group originating from the singly-bonded water. On the other hand, in propanediol-water mixtures these groups were not observed. ${ }^{18}$ The presence or absence of the free $\mathrm{OH}$ groups is of great importance for elucidation of the correct structure of the mixtures. NIR spectroscopy is a particularly useful tool for examination of the state of water since the $\nu_{2}+\nu_{3}$ band appears in the region free from absorption of the other functional groups. ${ }^{19,20}$ The absorptivities of NIR bands are much weaker than those in the IR spectra, and more convenient path lengths can be used. Besides, the bands originating from different hydrogen-bonded species are better separated in the overtone region. ${ }^{21-23}$

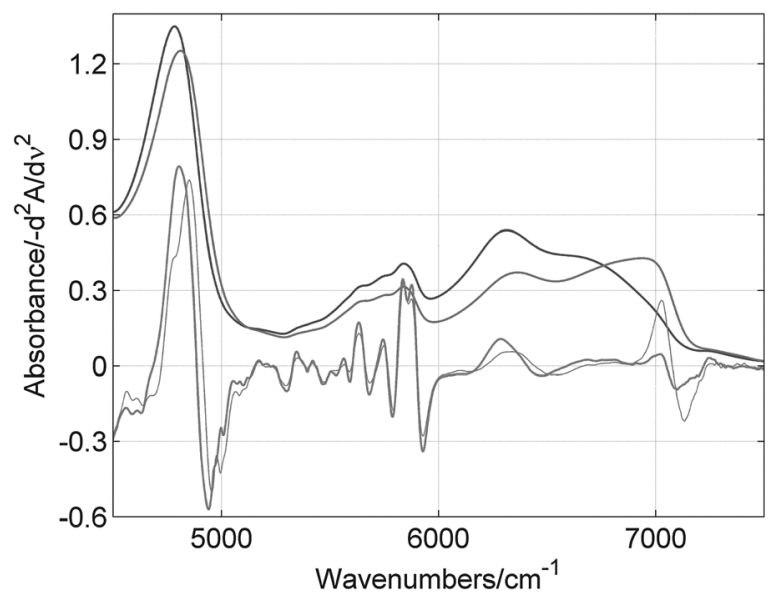

Fig. 2 NIR spectra of neat EG at $10{ }^{\circ} \mathrm{C}$ (blue) and $90{ }^{\circ} \mathrm{C}$ (red) together with the second derivative spectra at $10{ }^{\circ} \mathrm{C}$ (green) and $90{ }^{\circ} \mathrm{C}$ (magenta). To appear in this scale, the second derivative spectra were multiplied by 2000 .

Here we report studies on the effect of temperature and concentration on molecular structure and hydrogen bonding of EG and EG-water binary mixtures in the liquid phase. Particular attention was paid to the state of water in the mixture and the effect of water content on the structure of EG-water mixture. The structure of the liquid phase as well as the interaction between water and solvent determine the solubility of water in the organic phase. Hence, the comparison of the results for binary mixtures of water with various organic solvents is expected to provide new information on the relationship between the solubility and the chemical structure of the organic solvent. To enhance the spectral resolution and obtain more detailed information from NIR spectra we applied the generalized 2D correlation analysis, ${ }^{24-26}$ the moving window approach ${ }^{27}$ and chemometric methods like principal component analysis (PCA), ${ }^{28}$ evolving factor analysis $(\mathrm{EFA})^{29}$ and multivariate curve resolution-alternating least squares (MCR-ALS) analysis. ${ }^{30}$

\section{Experimental}

\section{Materials and methods}

EG (purity $>99.5 \%$ ) was purchased from Wako Pure Chemical Industries, Ltd (Osaka, Japan) and dried with activated molecular sieves ( 4 Å) before use. FT-NIR transmission spectra were recorded at a resolution of $4 \mathrm{~cm}^{-1}$ on a Perkin-Elmer Spectrum One NTS FT-NIR spectrometer equipped with a DTGS detector, and 256 scans were accumulated. NIR spectra were recorded in 1 or $0.3 \mathrm{~mm}$ quartz cells in the temperature range of $10-90{ }^{\circ} \mathrm{C}$ in steps of $5{ }^{\circ} \mathrm{C}$. During the measurements the sample chamber was purged with dry nitrogen.

\section{D correlation and moving-window analysis}

At first the baseline fluctuations were reduced by an offset at $9000 \mathrm{~cm}^{-1}$, and then the spectra were normalized against the density or concentration changes. ${ }^{31}$ The generalized $2 \mathrm{D}$ correlation spectra were calculated according to Noda's algorithm, ${ }^{24,25}$ using MATLAB 7.0.4 (The Math Works Inc.) based software. ${ }^{20}$ 
The spectrum of pure EG at $25{ }^{\circ} \mathrm{C}$ and the temperature-average spectrum were used as references for the data recorded as a function of water content and temperature, respectively. The detailed properties of the asynchronous spectra are explained elsewhere $;^{24-26}$ here we briefly summarize only the most important features. The asynchronous spectrum indicates how much the peaks at two different wavenumbers are unlike each other. For easy interpretation of the asynchronous spectra they were multiplied by the sign of the corresponding synchronous spectra. According to this a positive asynchronous cross-peak at $\left(\nu_{i}, \nu_{j}\right)$ means that the spectral change at $\nu_{i}$ occurs earlier/ faster than that at $\nu_{j}$, while negative peaks indicate the opposite. The asynchronous spectrum develops a cross-peak at $\left(\nu_{i}, \nu_{j}\right)$ if the spectral responses at $\nu_{i}$ and $\nu_{j}$ vary out of phase for some values of the perturbation or follow different response functions. The presence of the asynchronous peak indicates that the spectral responses at $\nu_{i}$ and $\nu_{j}$ originate from different molecules or distinct parts of the same molecule responding in a different way to the applied perturbation.

The moving-window 2D correlation analysis was performed following the method proposed by Thomas and Richardson, ${ }^{27}$ and the window length was set to three spectra. This method is based on the partitioning of the entire data set into smaller subsets (windows) and sequentially calculating 2D correlation spectra for each of the data subsets. Next, the diagonal of the synchronous spectra (power spectrum) are plotted versus the average temperature of the window. The power spectrum represents the overall extent of intensity changes at individual wavenumbers. In this way the moving-window 2D correlation analysis yields detailed information on the spectral changes at particular wavenumbers as a function of the temperature.

\section{Chemometric analysis}

The number of major species in each data set was obtained by PCA, and then verified by EFA. The initial concentration profiles were estimated from EFA. The real concentration profiles and the pure component spectra were resolved using the MCR-ALS method with constraints (non-negativity of concentrations and spectra). The main purpose of MCR-ALS is resolution of spectra of pure components $\left(S_{i}\right)$ and the corresponding concentration profiles $\left(C_{i}\right)$ from the overlapping spectra of the mixtures $(A):^{30}$

$$
A=\sum_{i}^{N} C_{i} \cdot S_{i}^{T}+E
$$

where the superscript $T$ means transpose, $N$ is the number of the components present in the system and $E$ is the residual matrix. The spectral profiles have chemical significance and are proportional to the spectra of pure constituents, while the concentration profiles are equal to the real concentrations up to a normalization factor. The chemometric analysis was performed using PLS-Toolbox 6.2 (Eigenvector Research Inc.) for MATLAB.

\section{Second derivative spectra}

The second derivative spectra were calculated using the savgol function included in PLS-Toolbox. The window size was 15 or 21 points and the polynomial degree was set to 2 .

\section{DFT calculations}

To clarify the local bonding structure the structures of EG and two EG-water complexes ( $4: 1$ and $4: 2)$ were optimized using the density functional theory (DFT) method. DFT calculations were performed using Gaussian 03W at the B3LYP/6-311++G(d,p) level of theory. ${ }^{32}$ The total energy of each structure was corrected using the zero point vibrational energy, and the harmonic frequencies were calculated. All stationary points were found to be true minima since no imaginary frequencies were obtained.

\section{Results and discussion}

\section{Effect of temperature on NIR spectra of neat EG and EG-water equimolar mixtures}

Fig. 2 shows NIR and second derivative spectra of liquid EG at 10 and $90{ }^{\circ} \mathrm{C}$. The corresponding peak positions are collected in Table 1 . The broad band near $6900 \mathrm{~cm}^{-1}$ (weakly bonded $\mathrm{OH}$ ) obscures a high frequency component at $7030 \mathrm{~cm}^{-1}$ (free $\mathrm{OH}$ ) that was resolved in the second derivative spectra. The intensity of this band increases upon temperature rise from 10 to $90{ }^{\circ} \mathrm{C}$, whereas its position is nearly the same $\left(\Delta \nu=8 \mathrm{~cm}^{-1}\right)$. In contrast, the band near $6300 \mathrm{~cm}^{-1}$ (strongly bonded $\mathrm{OH}$ ) decreases

Table 1 Assignments of NIR bands of EG and EG-water mixture in the liquid phase

\begin{tabular}{|c|c|c|}
\hline Vibration & Position $\left[\mathrm{cm}^{-1}\right]$ & Assignment \\
\hline \multicolumn{3}{|c|}{ Bulk EG at $10 / 90{ }^{\circ} \mathrm{C}$} \\
\hline $2 \nu(\mathrm{OH})$ & $6285 / 6339$ & EG-EG, strongly bonded \\
\hline $2 \nu(\mathrm{OH})$ & $-/ 6870$ & EG-EG, weakly bonded \\
\hline $2 \nu(\mathrm{OH})$ & $7022 / 7030$ & Free \\
\hline \multicolumn{3}{|c|}{ EG-water mixture $(X=0.5)$ at $10 / 90{ }^{\circ} \mathrm{C}$} \\
\hline$\nu_{2}+\nu_{3}$ & $5164 / 5207$ & EG- $\mathrm{H}_{2} \mathrm{O}-\mathrm{EG}+\mathrm{H}_{2} \mathrm{O}-\mathrm{H}_{2} \mathrm{O}, \mathrm{H}_{2} \mathrm{O}-\mathrm{H}_{2} \mathrm{O}$, bonded \\
\hline$\nu_{1}+\nu_{3}$ & $6846 / 6981$ & $\mathrm{EG}-\mathrm{H}_{2} \mathrm{O}-\mathrm{EG}+\mathrm{H}_{2} \mathrm{O}-\mathrm{H}_{2} \mathrm{O}, \mathrm{H}_{2} \mathrm{O}-\mathrm{H}_{2} \mathrm{O}$, bonded \\
\hline \multicolumn{3}{|c|}{ EG-water mixture with a water content of $5 \% / 50 \% / 95 \%$ at $25{ }^{\circ} \mathrm{C}$} \\
\hline$\nu_{2}+\nu_{3}$ & $5149 / 5166 / 5182$ & EG- $\mathrm{H}_{2} \mathrm{O}-\mathrm{EG}, \mathrm{H}_{2} \mathrm{O}-\mathrm{H}_{2} \mathrm{O}$, bonded \\
\hline $2 \nu(\mathrm{OH})$ & $6273 / 6273 / 6275$ & EG-EG, bonded \\
\hline$\nu_{1}+\nu_{3}$ & $-/ 6850 / 6881$ & EG- $\mathrm{H}_{2} \mathrm{O}-\mathrm{EG}, \mathrm{H}_{2} \mathrm{O}-\mathrm{H}_{2} \mathrm{O}$, bonded \\
\hline
\end{tabular}




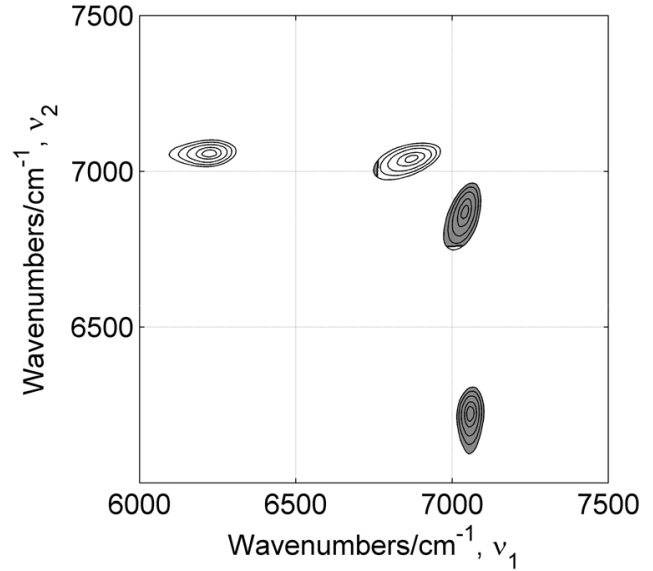

Fig. 3 Asynchronous 2D correlation spectrum of neat EG from 10 to $90{ }^{\circ} \mathrm{C}$. Positive peaks were shaded.

in intensity and is appreciably blue-shifted $\left(\Delta \nu=54 \mathrm{~cm}^{-1}\right)$ upon temperature increase, suggesting reduction in the extent and strength of the hydrogen bonding. Additional information on the molecular mechanism of these changes was provided by 2D correlation analysis. The asynchronous spectrum (Fig. 3) develops two peaks correlating three different wavenumbers $\left(6225,6870\right.$ and $\left.7050 \mathrm{~cm}^{-1}\right)$. The 2D correlation peaks are usually shifted with respect to the real peaks. ${ }^{33-35}$ Thus, the asynchronous peaks at 6225 and $7050 \mathrm{~cm}^{-1}$ represent the spectral changes at $6300 \mathrm{~cm}^{-1}$ and $7030 \mathrm{~cm}^{-1}$, respectively. In the asynchronous spectrum an additional peak at $6870 \mathrm{~cm}^{-1}$ assigned to the weakly bonded $\mathrm{OH}$ was also resolved. This $2 \mathrm{D}$ correlation peak corresponds to that at $6900 \mathrm{~cm}^{-1}$ observed in NIR spectrum of EG at $90{ }^{\circ} \mathrm{C}$. Both the 6225 and $6870 \mathrm{~cm}^{-1}$ peaks originate from the intermolecular interactions. ${ }^{18,20}$ This means that in the spectrum of bulk EG the peak due to the intramolecular hydrogen bond does not appear. The strongly bonded $\mathrm{OH}$ groups are both donors and acceptors in the higher associates, where the hydrogen bonding is significantly strengthened due to the cooperativity effect. In contrast, in the small associates this effect does not occur and as a result the hydrogen bonding is much weaker. In this case, even an acceptance of another bond does not significantly change the strength of the donor. Hence, the weakly hydrogen bonded $\mathrm{OH}$ groups can be both the donors and the donors and acceptors simultaneously in the small associates. The sign of the asynchronous peaks reveals that the spectral changes at $7050 \mathrm{~cm}^{-1}$ occur faster (at lower temperature) than those at 6225 and $6870 \mathrm{~cm}^{-1}$. As can be seen from the moving window analysis (Fig. 4) the changes of the free and associated $\mathrm{OH}$ groups have distinct patterns. The maximum of spectral changes for the free $\mathrm{OH}$ appears at $40{ }^{\circ} \mathrm{C}$, whereas for the associated $\mathrm{OH}$, at $50{ }^{\circ} \mathrm{C}$.

Fig. 5 shows the NIR spectra of the EG-water equimolar mixture and bulk water at 10 and $90{ }^{\circ} \mathrm{C}$. The temperature rise shifts the position of the $\nu_{2}+\nu_{3}$ band of water in the mixture from 5164 to $5207 \mathrm{~cm}^{-1}$, while the corresponding band of bulk water is shifted from 5175 to $5228 \mathrm{~cm}^{-1}$. A similar trend was observed for the $\nu_{1}+\nu_{3}$ band of water in the mixture that shifts

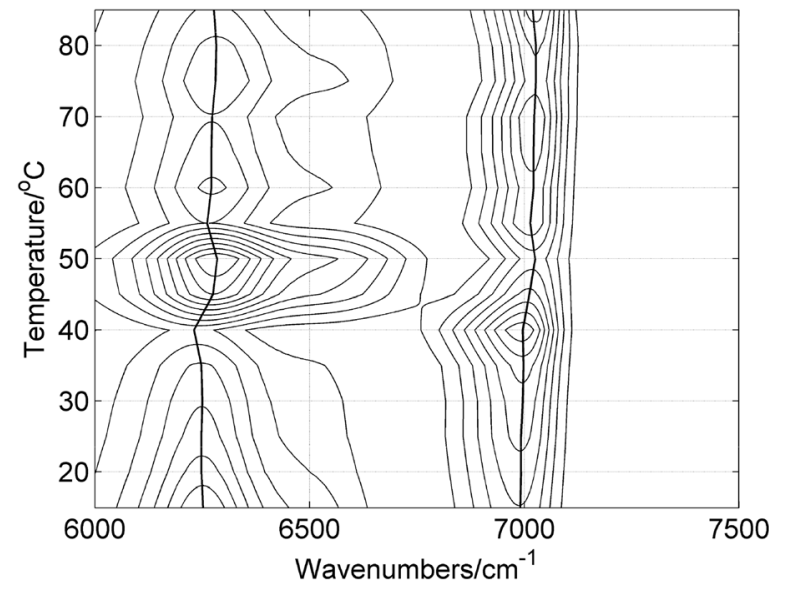

Fig. 4 Moving-window 2D correlation spectrum of neat EG from 10 to $90^{\circ} \mathrm{C}$.

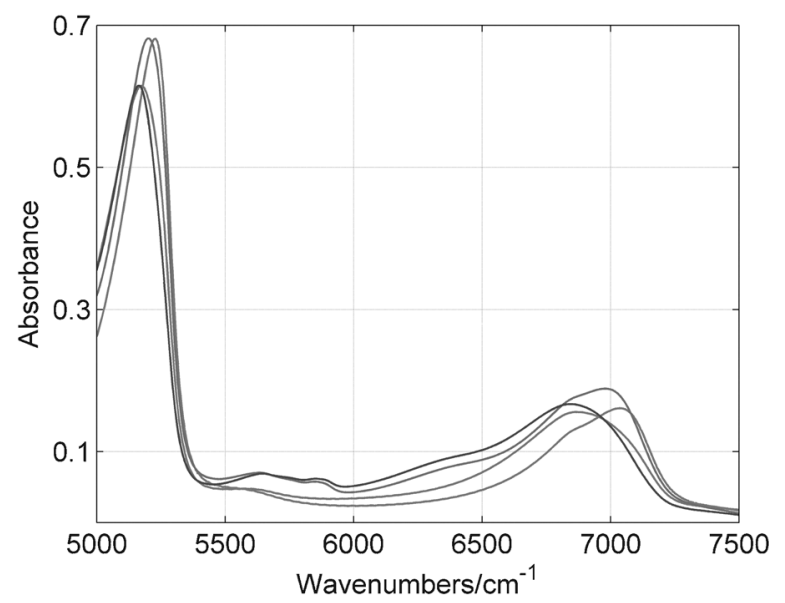

Fig. $5 \mathrm{NIR}$ spectra of water in EG-water equimolar mixture at $10^{\circ} \mathrm{C}$ (blue) and $90^{\circ} \mathrm{C}$ (red) together with the spectra of bulk water at $10^{\circ} \mathrm{C}$ (magenta) and $90{ }^{\circ} \mathrm{C}$ (green). The spectra of bulk water were normalized against the intensities of the spectra of water in the mixture.

from $6850 \mathrm{~cm}^{-1}$ to $6981 \mathrm{~cm}^{-1}$ upon temperature rise from 10 to $90^{\circ} \mathrm{C}$. For comparison, in bulk water this band shifts from 6869 to $7040 \mathrm{~cm}^{-1}$. Clearly, in this temperature range the molecules of water in the equimolar mixture are involved in stronger hydrogen bonding as compared with those in bulk water, and this difference is more distinct at higher temperatures. This means that water-water hydrogen bonding weakens faster than that of EG-water upon temperature rise. The results obtained for the mixtures of water with propanediols ${ }^{18}$ and aliphatic alcohols ${ }^{20,36}$ also reveal that the interaction between water and diol or alcohol is stronger than that of water-water. The $\nu_{2}+\nu_{3}$ band of water has a high frequency component that was resolved in the second derivative spectra (not shown). At elevated temperatures this band has the same position $\left(5255 \mathrm{~cm}^{-1}\right)$ both in the mixture and in bulk water, suggesting that the temperature increase leads to the formation of similar species in both systems. From the position of this peak one can conclude that the water-water interactions within these clusters are relatively weak. The moving window 2D correlation analysis (Fig. 6) reveals 


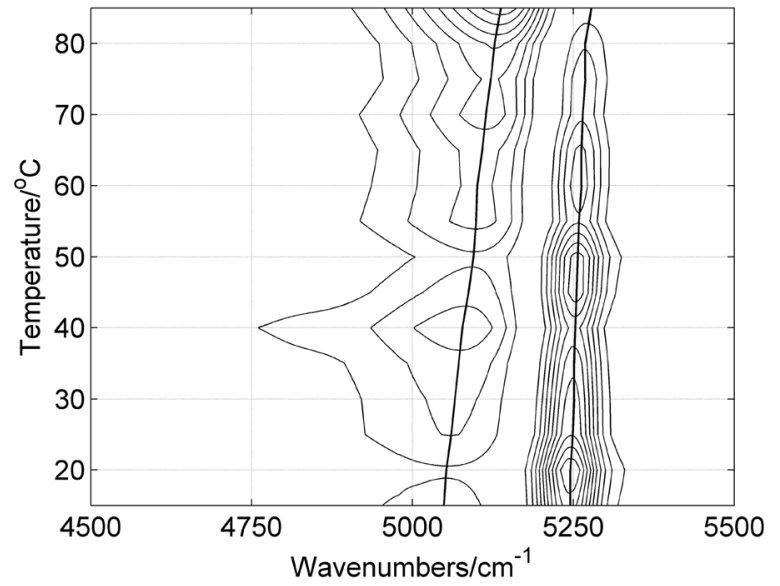

Fig. 6 Moving-window 2D correlation spectrum of EG-water equimolar mixture from 10 to $90{ }^{\circ} \mathrm{C}$.

that the high frequency band of water is only slightly shifted with the temperature rise, whereas the low frequency band reveals a more distinct shift. Besides, the extent of spectral changes for strongly bonded species increases with the temperature, whereas for weakly bonded water one can observe the opposite tendency. This means that the thermal disruption of strongly bonded species appears more easily at higher temperatures. In contrast, the population of weakly hydrogen bonded species increases predominantly at lower to moderate temperatures.

\section{Effects of water content on NIR spectra of EG-water mixtures}

NIR spectra of EG-water mixtures (Fig. 7) were recorded from pure EG to pure water in steps of $5 \%$. In the spectra dominates strong absorption from water, whereas the absorption from EG is hardly seen. In the water-poor region the $\nu_{1}+\nu_{3}$ band is overlapped by the $\mathrm{OH}$ absorption from EG, and therefore more reliable information on the state of water provides the $\nu_{2}+\nu_{3}$ band. The position of this band shifts from $5149 \mathrm{~cm}^{-1}$ in diluted solution $(5 \%)$ to $5166 \mathrm{~cm}^{-1}$ for the $50 \%$ water content one, and reaches $5182 \mathrm{~cm}^{-1}$ in bulk water. At the same time, the $\nu_{1}+\nu_{3}$

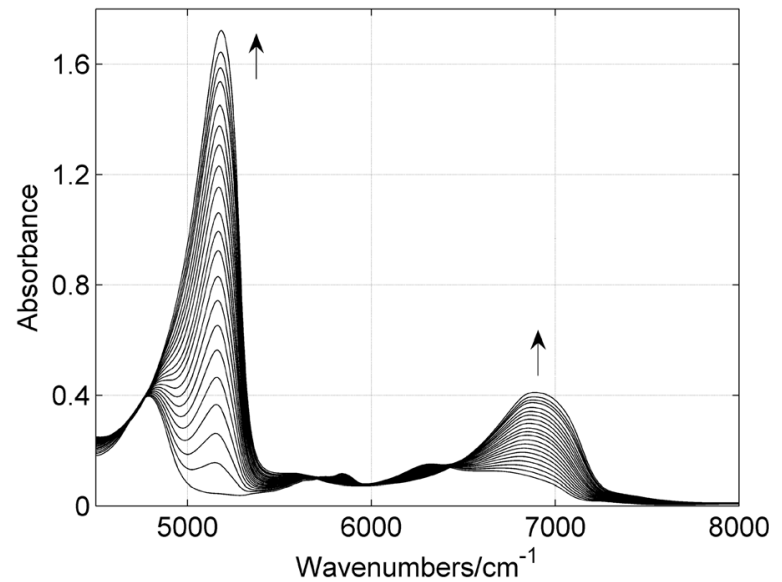

Fig. 7 NIR spectra of EG-water mixture from pure EG to pure water with a step of $5 \%$ (weight) at $25{ }^{\circ} \mathrm{C}$. The arrows indicate the direction of increasing water content. band shifts from $6850 \mathrm{~cm}^{-1}(50 \%)$ to $6887 \mathrm{~cm}^{-1}$ (bulk water). This confirms our earlier observation that the molecules of water in the mixture participate in stronger hydrogen bonding than those in bulk water. It is also clear that an increase in the water content in the mixture weakens the average strength of hydrogen bonding of water, as a result of a growing number of water clusters.

The second derivative spectrum (Fig. 8a) reveals that the $\nu_{2}+\nu_{3}$ band has two components. The position of the higher frequency band $\left(5253 \mathrm{~cm}^{-1}\right)$ does not vary with the water content, and is the same as that in the second derivative spectrum of bulk water. In contrast, the low frequency band is blue-shifted from 5162 to $5191 \mathrm{~cm}^{-1}\left(\Delta \nu=29 \mathrm{~cm}^{-1}\right)$. Also in the asynchronous spectrum constructed from the spectra of the mixtures (Fig. 8b) the $\nu_{2}+\nu_{3}$ band is resolved into two components located at 5149 and $5255 \mathrm{~cm}^{-1}$. As can be seen (Fig. 8b), similar splitting occurs for the $\nu_{1}+\nu_{3}$ band. The lower frequency band was assigned to water involved in the cooperative interaction with EG, while the other bands result from water-water interactions. Hence, it is clear from Fig. 8a that increasing water content leads to growth
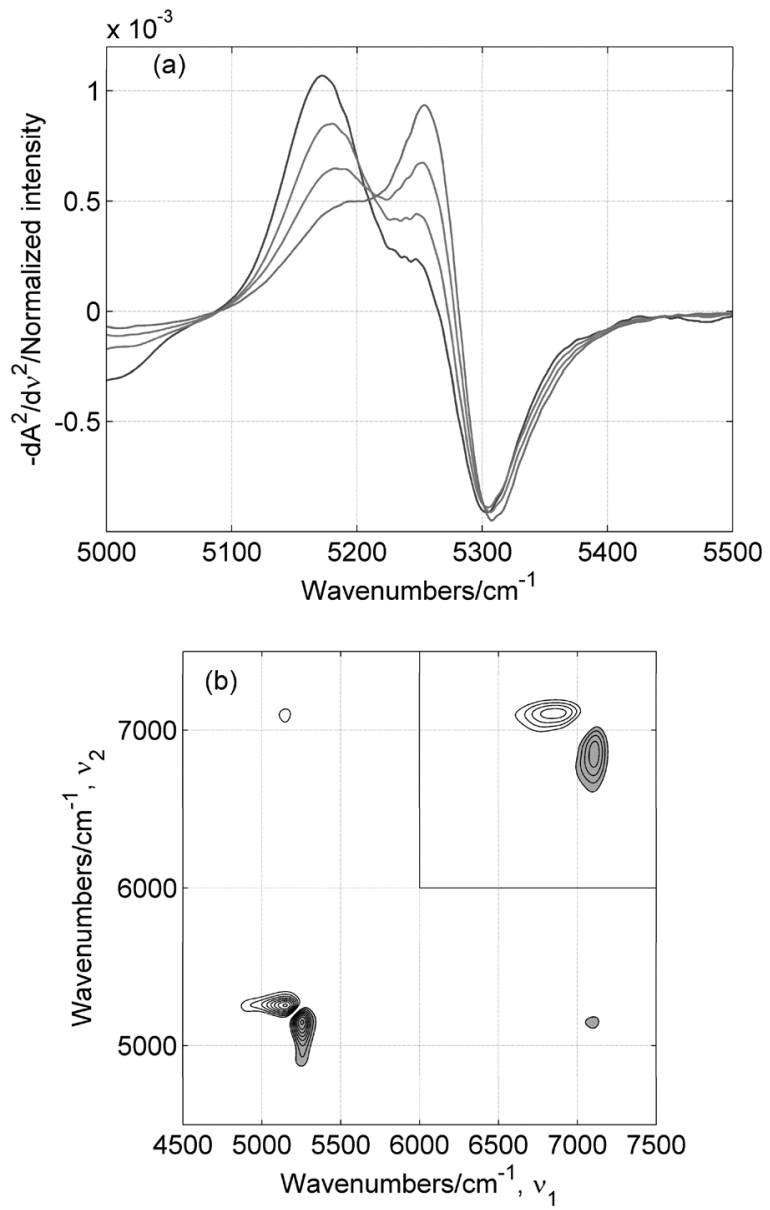

Fig. 8 Normalized (against water content) second derivative spectra of EG-water mixtures at a water content of 25\% (blue), 50\% (magenta), 75\% (green) and $100 \%$ (red) (a). The asynchronous 2D correlation spectrum (b) constructed from the spectra of EG-water mixtures shown in Fig. 7. Positive asynchronous peaks were shaded. 
in the population of the clusters of water, partially at the expense of the EG-water species.

PCA and EFA analysis (not shown) suggest the presence of three independent components contributing to the concentrationdependent changes in the spectra of EG-water mixtures. Fig. 9 displays the concentration and spectral profiles obtained from MCR-ALS. For this purpose we used the als function from PLSToolbox and the initial concentration profiles were estimated from EFA (evolvfa function). The spectral component decreasing in intensity is very similar to the spectrum of pure EG, and was assigned to EG. This similarity confirms our earlier conclusion that addition of water has a small effect on the structure of organic phase. ${ }^{18,20,36}$ Recently, analogous result was obtained from neutron diffraction studies of glycerol-water mixtures. ${ }^{37}$ It was shown that glycerol-glycerol hydrogen bonding was largely unperturbed by the presence of water in the mixture. The spectral profile of the component increasing in intensity (Fig. 9a) is the same as that of the spectrum of bulk water. Therefore, this component was assigned to water involved in water-water interactions. The third component at first increases in intensity up to about $50 \%$, and then decreases to zero intensity in pure water (Fig. 9a). The $\nu_{2}+\nu_{3}$ combination band
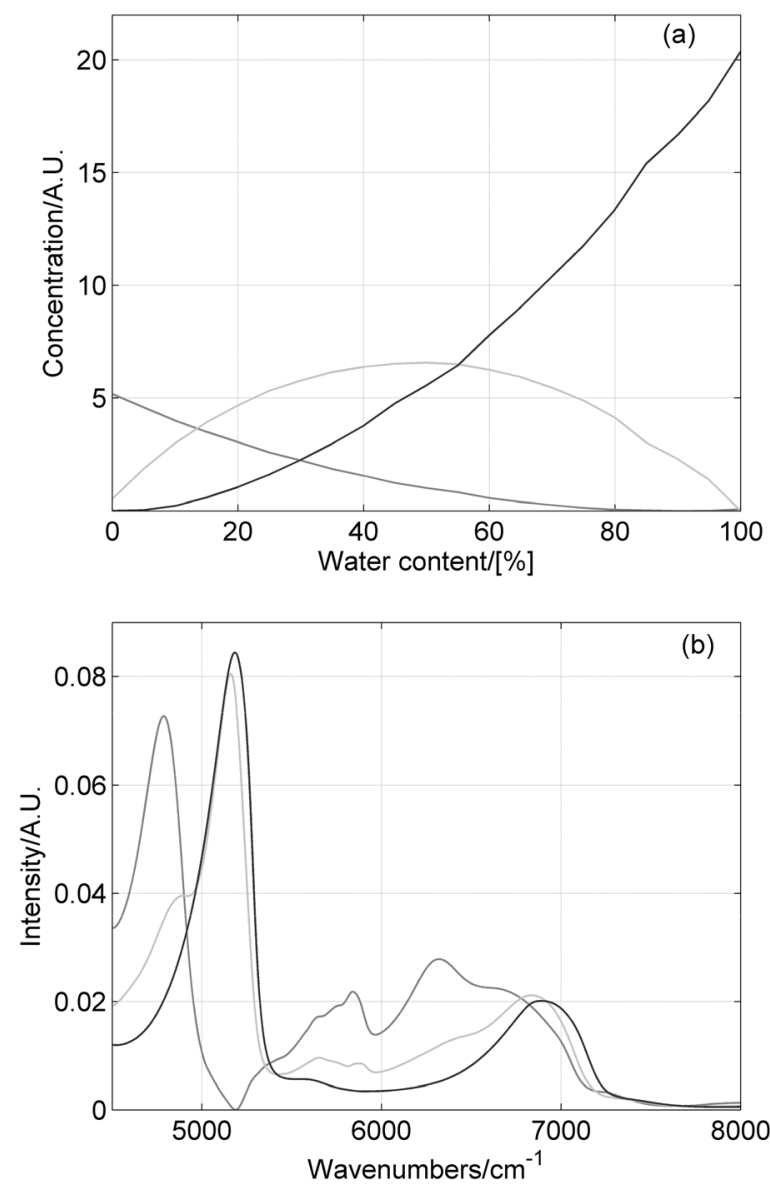

Fig. 9 Concentration profiles of water (blue, green) and EG (red) (a) together with the corresponding spectral profiles of water (blue, green) and EG (red) (b) obtained from MCR-ALS of the concentration-dependent spectra of EG-water mixture at $25^{\circ} \mathrm{C}$. in this spectral profile (Fig. 9b) is $22 \mathrm{~cm}^{-1}$ red-shifted as compared with the corresponding band in the spectrum of bulk water evidencing that this component participates in stronger interactions than those in bulk water. Hence, this spectral profile was assigned to water engaged in interaction with EG. The spectral changes for both components of water are unlike, giving rise to the asynchronicity observed in Fig. 8b.

\section{Structure of EG-water mixtures}

The spectra of alcohol-water mixtures reveal the presence of singlybonded water with the free $\mathrm{OH}$ absorbing at $5300 \mathrm{~cm}^{-1} \cdot{ }^{20,36}$ On the other hand, in the mixture of EG or propanediols ${ }^{18}$ with water the free $\mathrm{OH}$ was not observed. This suggests that the state of water in diols is different from that in the aliphatic alcohols. From Fig. 9a it is clear that in the water-poor region (water content $<50 \%$ ) EG-water interactions are preferred over waterwater ones. Since the free $\mathrm{OH}$ groups of water do not appear in the mixture, one can conclude that the molecules of water are double donors to two molecules of EG. An exemplary structure of this complex obtained from DFT calculations is shown in Fig. 10a. In the water-poor region the molecules of water predominantly do not interact with the other molecules of water and mostly appear as monomers, as was suggested by MD studies. ${ }^{38,39}$ Due to the large extent of self-association and very high viscosity, the structure of liquid diols is more stable as compared with
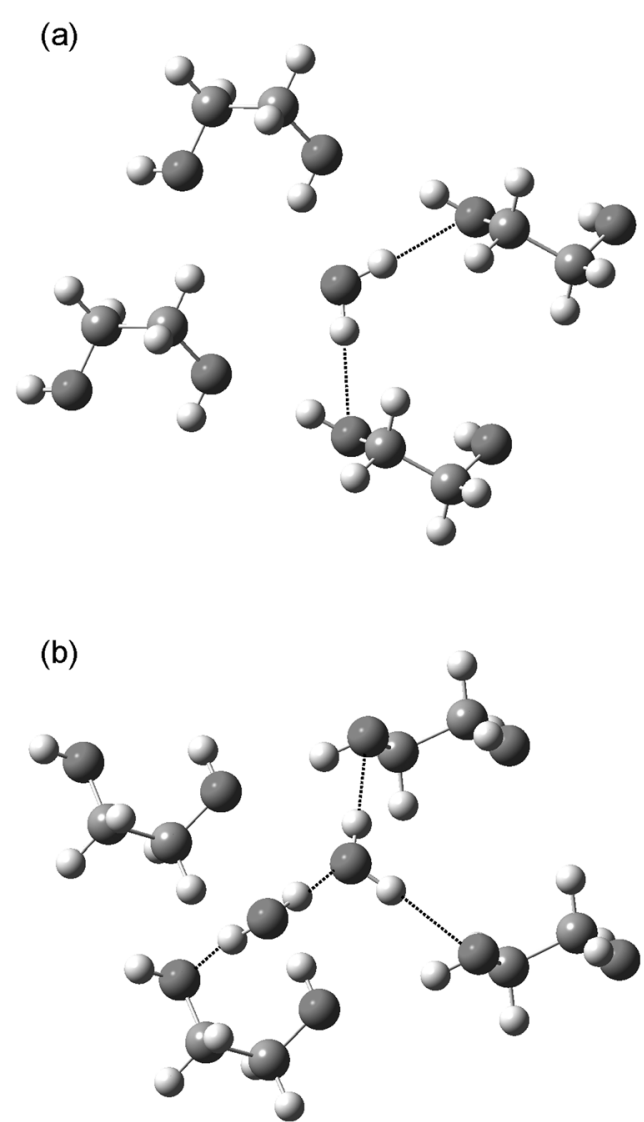

Fig. 10 Optimized structures of 4:1 (a) and 4:2 (b) EG-water complexes obtained from DFT calculations. 
that of the aliphatic alcohols. Thus, the free $\mathrm{OH}$ groups of water do not appear in the mixture with diols. Higher water content increases the probability of water-water interaction, leading to the formation of water clusters around the OH groups of EG. The $5255 \mathrm{~cm}^{-1}$ band originates from absorption of weakly hydrogenbonded water in these clusters. The optimized structure of the cluster consisting of two molecules of water is displayed in Fig. 10b. As can be seen, in small clusters the molecules of water may interact both with the other molecules of water and with the molecules of EG. The growing size of these clusters is expected to increase the number of water-water hydrogen bonds at the expense of EG-water ones. This supposition is well illustrated in Fig. 8a.

A MD study has shown that the structure of liquid EG is dominated by three-dimensional networks of hydrogen bonded molecules. ${ }^{39}$ This results from the fact that each molecule of EG can act as a double proton donor and a double proton acceptor, forming up to four hydrogen bonds. The presence of the threedimensional structure in liquid EG leads to relatively homogeneous distribution of the $\mathrm{OH}$ groups through the entire volume. This forces a homogeneous distribution of water forming small clusters around $\mathrm{OH}$ groups of EG. As a result, water is fully miscible with EG. The size of water clusters depends on the concentration of EG: the higher EG concentration the smaller the water clusters. This conclusion is supported by a MD study of glycerol-water liquid mixtures showing that the increase of glycerol content causes reduction in the size of water clusters. ${ }^{40}$

Dielectric measurements of the short chain alcohols, from methanol to propanol, demonstrated the preference for formation of long chain linear associates in the liquid phase. ${ }^{41}$ This causes less homogeneous distribution of the $\mathrm{OH}$ groups as compared with liquid EG, and the molecules of water may form larger clusters. The water-water-interaction in these clusters is stronger as compared with that in the EG-water mixture, and hence the $\nu_{2}+\nu_{3}$ band of water in alcohols occurs near $5200 \mathrm{~cm}^{-1} \cdot{ }^{20,36}$ For comparison, the corresponding band in bulk water is red-shifted only by $16 \mathrm{~cm}^{-1}$. Still the alcohol-water interaction is strong enough to prevent the phase separation. The interaction of the hydrophobic parts has a minor impact on the structure of short chain alcohols in the liquid phase. A neutron diffraction study of $7: 3$ mole ratio methanol-water solution has shown that the properties of this mixture are influenced mainly by the polar interaction of water with the $\mathrm{OH}$ group of methanol. ${ }^{42}$ In longer chain alcohols $(n>3)$ there is significant increase of the hydrophobic interactions, and the tendency for creation of the micelle-like associates. ${ }^{43}$ As a result, the distribution of the $\mathrm{OH}$ groups in the liquid phase is not uniform, and the interaction between molecules of water and these associates is less favourable. Thus, the molecules of water tend to form large clusters, and the alcohol-water interaction is too weak to prevent the phase separation. Branching in the vicinity of the $\mathrm{OH}$ group reduces the extent of self-association of alcohol. The associates of these alcohols are smaller and the $\mathrm{OH}$ groups are easier to access for interaction with water. Besides, the interaction between the hydrophobic parts of tert-butyl alcohol is weaker than that in $n$-butyl alcohol. As a result, the solubility of water in tert-butyl alcohol is unlimited, whereas in $n$-butanol it is limited. It seems that the free $\mathrm{OH}$ groups of alcohols or other solvents do not decide on the extent of solubility of water in the organic phase. Also the presence or absence of the intramolecular bonding has a minor impact on the structure of the condensed phase, as concluded from MD studies. ${ }^{37}$ Comparing the present results with those previously obtained for binary mixtures of water with different organic solvents ${ }^{20,36}$ one can conclude that the solubility of water in the organic phase is determined primarily by the total number and distribution of the polar groups. In turn, the distribution of these groups in the organic phase depends on the size and structure of the hydrocarbon chain. This suggestion was confirmed using ${ }^{17} \mathrm{O}$ NMR studies of dynamic hydration of diols and alcohols in aqueous solutions. ${ }^{44}$

\section{Conclusions}

Molecules of EG in the pure liquid phase participate in the intermolecular hydrogen bonding, while the intramolecular bonds were not observed. In water-poor regions EG-water interactions are preferred over water-water ones. The molecules of water are doubly-bonded to two different molecules of EG and this cooperative interaction is stronger than that in bulk water. An increase in the water content weakens the average strength of the hydrogen bonding of water but has a small effect on the structure of liquid EG. Molecules of water form clusters located around $\mathrm{OH}$ groups of EG. The size of these clusters depends on the concentration of EG, the smaller concentration of EG, the larger the size of water clusters. The comparison of the present results with previous studies on binary mixtures of water with aliphatic alcohols and diols suggests that the solubility of water in the binary mixture is determined primarily by the total amount and distribution of the functional groups in the organic phase. The larger the population and more uniform distribution of these groups, the better the solubility of water in the organic phase. The distribution of the functional groups in the liquid phase depends on the size and structure of the hydrophobic part of the molecule.

\section{References}

1 R. A. Klein, J. Comput. Chem., 2002, 23, 585-599.

2 R. A. Klein, Chem. Phys. Lett., 2006, 429, 633-637.

3 M. Mandado, A. M. Graña and R. A. Mosquera, Phys. Chem. Chem. Phys., 2004, 6, 4391-4396.

4 D. Chopra, T. N. G. Row, E. Arunan and R. A. Klein, J. Mol. Struct., 2010, 964, 126-133.

5 L. Weng, C. Chen, J. Zuo and W. Li, J. Phys. Chem. A, 2011, 115, 4729-4737.

6 S. Malekina, B. Friedman, N. Abedi and M. Schwartz, Spectrosc. Lett., 1980, 13, 777-784.

7 D. L. Howard, P. Jørgensen and H. G. Kjaergaard, J. Am. Chem. Soc., 2005, 127, 17096-17103.

8 P. Bultinck, A. Goeminne and D. V. Vondel, THEOCHEM, 1995, 357, 19-32.

9 K. Takahashi, Phys. Chem. Chem. Phys., 2010, 12, 13950-13961. 
10 P. Manivet and M. Massela, Chem. Phys. Lett., 1998, 288, 642-646.

11 D. L. Crittenden, K. C. Thomopson and J. T. Jordan, J. Phys. Chem. A, 2005, 109, 2971-2977.

12 A. Chaudhari and S.-L. Lee, J. Chem. Phys., 2004, 120, 7464-7469.

13 C. J. Cramer and D. G. Truhlar, J. Am. Chem. Soc., 1994, 116, 3892-3900.

14 V. Crupi, G. Maisano, D. Majolino, P. Migliardo and V. Venuti, J. Phys. Chem. A, 2000, 104, 3933-3939.

15 R. M. Kumar, P. Baskar, K. Balamurugan, S. Das and V. Subramanian, J. Phys. Chem. A, 2012, 116, 4239-4247.

16 A. J. L. Jesus, M. T. S. Rosado, M. L. P. Leitão and J. S. Redinha, J. Phys. Chem. A, 2003, 107, 3891-3897.

17 D. L. Howard and H. G. Kjaergaard, J. Phys. Chem. A, 2006, 110, 10245-10250.

18 K. Z. Haufa and M. A. Czarnecki, Vib. Spectrosc., 2009, 51, 80-85.

19 B. Dickens and S. H. Dickens, J. Res. Natl. Inst. Stand. Technol., 1999, 104, 173-183.

20 M. A. Czarnecki, Appl. Spectrosc. Rev., 2011, 46, 67-103.

21 H. W. Siesler, Y. Ozaki, S. Kawata and H. M. Heise, NearInfrared Spectroscopy: Principles, Instruments, Applications, Wiley-VCH, Weinheim, Germany, 2002.

22 “Handbook of Near-Infrared Spectroscopy", ed. D. A. Burns and E. W. Ciurczak, CRC Press, Boca Raton, FL, 3rd edn, 2008.

23 M. A. Czarnecki, Y. Liu, Y. Ozaki, M. Suzuki and M. Iwahashi, Appl. Spectrosc., 1993, 47, 2162-2168.

24 I. Noda, Appl. Spectrosc., 1993, 47, 1329-1336.

25 I. Noda, Appl. Spectrosc., 2000, 54, 994-999.

26 I. Noda and Y. Ozaki, Two-Dimensional Correlation Spectroscopy: Applications in Vibrational Spectroscopy, John Wiley \& Sons, New York, 2004.

27 M. Thomas and H. H. Richardson, Vib. Spectrosc., 2000, 24, 137-146.

28 S. Wold, K. Esbensen and P. Geladi, Chemom. Intell. Lab. Syst., 1987, 2, 37-52.

29 H. R. Keller and D. L. Massart, Chemom. Intell. Lab. Syst., 1992, 12, 209-224.

30 R. Tauler, B. Kowalski and S. Fleming, Anal. Chem., 1993, 65, 2040-2047.
31 M. A. Czarnecki, Appl. Spectrosc., 1999, 53, 1392-1397.

32 M. J. Frisch, G. W. Trucks, H. B. Schlegel, G. E. Scuseria, M. A. Robb, J. R. Cheeseman, J. A. Montgomery, Jr., T. Vreven, K. N. Kudin, J. C. Burant, J. M. Millam, S. S. Iyengar, J. Tomasi, V. Barone, B. Mennucci, M. Cossi, G. Scalmani, N. Rega, G. A. Petersson, H. Nakatsuji, M. Hada, M. Ehara, K. Toyota, R. Fukuda, J. Hasegawa, M. Ishida, T. Nakajima, Y. Honda, O. Kitao, H. Nakai, M. Klene, X. Li, J. E. Knox, H. P. Hratchian, J. B. Cross, C. Adamo, J. Jaramillo, R. Gomperts, R. E. Stratmann, O. Yazyev, A. J. Austin, R. Cammi, C. Pomelli, J. W. Ochterski, P. Y. Ayala, K. Morokuma, G. A. Voth, P. Salvador, J. J. Dannenberg, V. G. Zakrzewski, S. Dapprich, A. D. Daniels, M. C. Strain, O. Farkas, D. K. Malick, A. D. Rabuck, K. Raghavachari, J. B. Foresman, J. V. Ortiz, Q. Cui, A. G. Baboul, S. Clifford, J. Cioslowski, B. B. Stefanov, G. Liu, A. Liashenko, P. Piskorz, I. Komaromi, R. L. Martin, D. J. Fox, T. Keith, M. A. Al-Laham, C. Y. Peng, A. Nanayakkara, M. Challacombe, P. M. W. Gill, B. Johnson, W. Chen, M. W. Wong, C. Gonzalez and J. A. Pople, Gaussian 03, Revision C.02, Gaussian, Inc., Wallingford CT, 2004.

33 A. Gericke, S. J. Gadaleta, J. W. Brauner and R. Mendelsohn, Biospectroscopy, 1996, 2, 341-361.

34 M. A. Czarnecki, Appl. Spectrosc., 1998, 52, 1583-1590.

35 M. A. Czarnecki, Appl. Spectrosc., 2000, 54, 986-993.

36 D. Wojtków and M. A. Czarnecki, J. Mol. Struct., 2008, 883-884, 203-208.

37 J. J. Towey, A. K. Soper and L. Dougan, J. Phys. Chem. B, 2011, 115, 7799-7807.

38 J. J. Towey, A. K. Soper and L. Dougan, J. Phys. Chem. B, 2012, 116, 13898-13904.

39 L. Saiz, J. A. Padró and E. Guàrdia, J. Chem. Phys., 2001, 114, 3187-3199.

40 A. V. Egorov, A. P. Lybartsev and A. Laaksonen, J. Phys. Chem. B, 2011, 115, 14572-14580.

41 P. Huyskens and P. Ruelle, J. Mol. Liq., 2000, 88, 87-108.

42 S. Dixit, J. Crain, W. C. K. Poon, J. L. Finney and A. K. Soper, Nature, 2002, 416, 829-832.

43 A. Vrhovšek, O. Gereben, A. Jamnik and L. Pusztai, J. Phys. Chem. B, 2011, 115, 13473-13487.

44 Y. Ishihara, S. Okouchi and H. Uedaira, J. Chem. Soc., Faraday Trans., 1997, 93, 3337-3342. 